\title{
An Analytical Development of the Hyperbolic Behaviour of Micro Thermoelectric Coolers
}

\author{
Giulia De Aloysio, ${ }^{1}$ Giampaolo D'Alessandro, ${ }^{2}$ and Filippo de Monte ${ }^{2}$ \\ ${ }^{1}$ Department of Information Engineering, Computer Science and Mathematics, University of L'Aquila, \\ Via Vetoio, Località Coppito, 67100 L'Aquila, Italy \\ ${ }^{2}$ Department of Industrial and Information Engineering and Economics, University of L'Aquila, \\ Via Giovanni Gronchi 18, 67100 L'Aquila, Italy
}

Correspondence should be addressed to Giulia De Aloysio; giulia.dealoysio@graduate.univaq.it

Received 9 March 2015; Revised 7 October 2015; Accepted 8 October 2015

Academic Editor: John D. Clayton

Copyright (C) 2015 Giulia De Aloysio et al. This is an open access article distributed under the Creative Commons Attribution License, which permits unrestricted use, distribution, and reproduction in any medium, provided the original work is properly cited.

\begin{abstract}
The transient behaviour of a micro thermoelectric cooler is described in the present paper through the theory of the thermal wave, involving the relaxation time. The internal heat generation due to the sudden application of the electric current is taken into account by means of the Heaviside function. The governing equations of both the semiconductors are solved by a modified Separation of Variables Method that allows us to have a better description of the device which can be obtained at early times. As regards the performances, the cooling load shows discontinuities due to the contributions of the travelling wave fronts of both the semiconductors. The results show that the coefficient of performance (COP) of the device obtained with the hyperbolic model is lower than that provided by the parabolic model at early times.
\end{abstract}

\section{Introduction}

In the literature many models related to the transient behaviour of thermoelectric devices have been presented [19]. These systems have large application as coolers [9-12], thanks to the possibility of maintaining the junction temperature as low as required. Most of the models make use of the parabolic thermal diffusion equation: three-dimensional numerical coupled models have been proposed recently, in which the coupling of the thermal and the electric field is considered, $[4,10]$. In particular, the coupling of both the fields is analyzed under unsteady and steady states for coolers in $[4,10]$.

However, the parabolic modelling is inadequate for devices with small dimensions, in particular in the analysis of their transient behaviour, during the start-up phase and the shut-down phase and when changing the operational parameters. In all of these cases it is more adequate to use the hyperbolic heat conduction model, which considers the relaxation time of the material [6-8]. This is a crucial parameter in the heat conduction theory because it takes into account the finite speed of the heat propagation. The relaxation time is absent in the classical theory of heat diffusion based on the Fourier law, whose intrinsic underlying assumption is that heat propagates in the material with an infinite speed.

Unlike the previously proposed model, in the present paper we describe the sudden application of the electric current by means of the Heaviside function. Following HajiSheikh and Beck's approach [13], the thermal field in both the semiconductors is obtained analytically by means of a modified Separation of Variables (SoV, for short) Method for time-independent boundary conditions of the first kind. According to this method, the solution of the hyperbolic problem is obtained by modifying the solution of the corresponding parabolic problem through an appropriate timedependent function. This function is due to the presence of the relaxation time and may be derived by imposing the modified solution which will be the solution of the hyperbolic problem. Then MATLAB ambient is used to implement the solution. Once the temperature distributions are known, the performances of the device, cooling load, and COP are evaluated. The results of the analysis show that the COP 


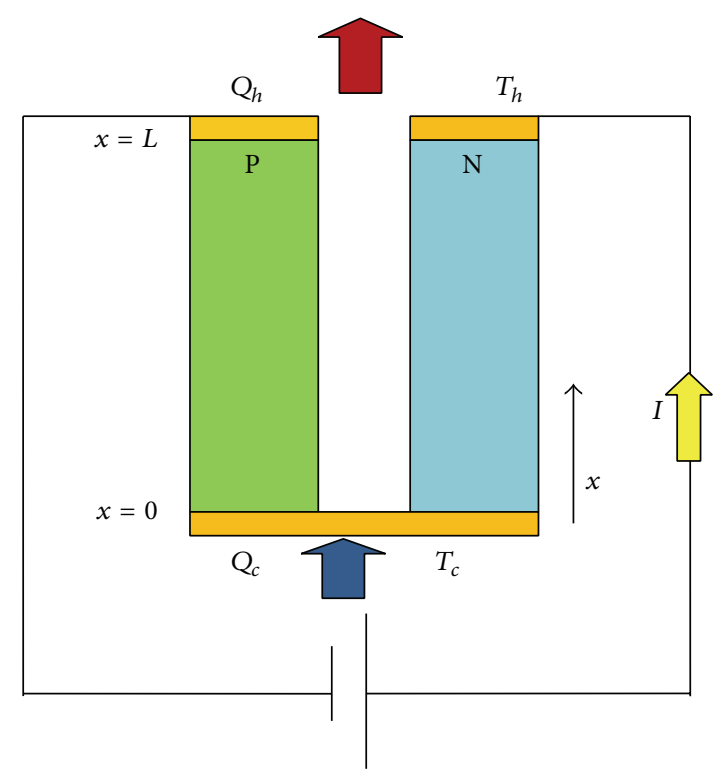

Figure 1: 1D schema of a thermoelectric cooler.

obtained by means of the hyperbolic model is lower than that provided by the parabolic model at early times.

\section{Analytical Formulation}

The elementary unit of a thermoelectric cooler (TEC) module consists of two semiconductors, $\mathrm{P}$ and $\mathrm{N}$, connected electrically in series, as shown in Figure 1. Because of the Peltier effect, by applying an electric current, heat is absorbed at the cold junction, whose temperature is denoted by $T_{c}$, and heat is released at the hot junction, whose temperature is $T_{h}$. The heat absorption at the cold junction is counteracted by Joule and Fourier's effects. It is worth noting that the Thomson effect is neglected in this analysis. The following assumptions are made to perform the analytical transient modelling of the device:

(a) The thermal conductivity and the electric resistivity are considered temperature-independent and computed at an average temperature $T_{\mathrm{avg}}=\left(T_{h}+T_{c}\right) / 2$, by using (23) (see ahead). This assumption is valid for low electric applied currents, as shown in [10].

(b) Adiabatic side surfaces of the TEC are taken into account.

(c) Semiconductors are employed in the analysis, which have the same geometrical characteristics, the crosssectional areas $A_{1}$ and $A_{2}$, and the lengths of the thermoelements, $L_{1}$ and $L_{2}$. We assume that $L_{1}=$ $L_{2}=L$.

(d) At the instant $t=0$ both the semiconductors have an ambient uniform temperature $T_{0}$ and a sudden constant uniform current is applied to the system.
2.1. Governing Equations for the Semiconductors. The energy equations for both the semiconductors are derived as suggested in [6]. However, in the present paper the energy equation is modified, as instantaneous internal heat generation has been considered by means of the Heaviside function in the hyperbolic model. Therefore, the energy balance equation valid for each semiconductor may be written as

$$
\rho c \frac{\partial T}{\partial t}+\frac{\partial q^{*}}{\partial x}+e J \frac{\partial \Phi}{\partial x} H(t)=0
$$

$H(t)$ represents the Heaviside function. In particular it is

$$
H(t)= \begin{cases}0 & \text { if } t<0 \\ 1 & \text { if } t>0\end{cases}
$$

The thermal flux at time $t+\bar{\tau}$ is defined as in (3a) as follows:

$$
q^{*}(x, t+\bar{\tau})=-k \frac{\partial T}{\partial x} \mp T S e J H(t)
$$

where $\bar{\tau}$ is the relaxation time and the minus sign stands for semiconductor $\mathrm{N}$, whereas the plus sign stands for semiconductor $\mathrm{P}$.

By applying Taylor expansion to (3a) and by neglecting the higher order terms, (3a) may be written as

$$
q^{*}(x, t)+\bar{\tau} \frac{\partial q^{*}}{\partial t}=-k \frac{\partial T}{\partial x} \mp T S e J H(t) .
$$

By combining (1) and ( $3 \mathrm{~b}$ ) and by performing some other calculations, as suggested in [6], the governing equation that allows us to determine the temperature distribution under 
the hyperbolic heat conduction model for both the pellets is given by (4a), (4b), and (4c). Consider

$$
\begin{aligned}
k A \frac{\partial^{2} T}{\partial x^{2}} & +\frac{R I^{2}}{L} H(t)-\rho A c \frac{\partial T}{\partial t}-\bar{\tau} \rho A c \frac{\partial^{2} T}{\partial t^{2}} \\
+ & \bar{\tau} \frac{\partial}{\partial t}\left(\frac{R I^{2}}{L} H(t) \mp S I \frac{\partial T}{\partial x} H(t)\right)=0 .
\end{aligned}
$$

Performing the derivative of the last term in (4a), a mixed derivative term should be generated. This term is neglected because its contribution is significantly lower than the one due to the other terms in (4a), if the length scale of the pellet is of the order of millimeters, as suggested in [8]. In order to solve (4a), initial and boundary conditions are provided in

$$
\begin{aligned}
T(x, 0) & =T_{0}, \\
\frac{\partial T}{\partial t}(x, 0) & =0, \\
T(0, t) & =T_{c}, \\
T(L, t) & =T_{h} .
\end{aligned}
$$

Before solving the problem, it is convenient to write it in dimensionless form as in [6], by introducing some dimensionless parameters, listed in the Nomenclature. Therefore, the dimensionless governing equation for both the semiconductors and the initial and the boundary conditions may be expressed by (5a), (5b) and (5c). Consider

$$
\begin{aligned}
& F_{i} \frac{\partial^{2} \vartheta_{i}}{\partial \xi^{2}}+D_{i}-\frac{\partial \vartheta_{i}}{\partial \eta}-\tau_{i} \frac{\partial^{2} \vartheta_{i}}{\partial \eta^{2}}+\tau_{i} D_{i} \delta(\eta)=0, \\
& \vartheta_{i}(\xi, 0)=0 \\
& \frac{\partial \vartheta_{i}}{\partial \eta}(\xi, 0)=0, \\
& \vartheta_{i}(0, \eta)=1, \\
& \vartheta_{i}(1, \eta)=\frac{T_{h}-T_{0}}{T_{c}-T_{0}}=h .
\end{aligned}
$$

The subscript $i$ indicates the type of the semiconductor; in fact $i=1$ is used for semiconductor $\mathrm{N}$, whereas $i=2$ is used for semiconductor P. The dimensionless groups $D_{i}$ and $F_{i}$ are defined as

$$
\begin{aligned}
& D_{1}=\frac{L R_{1} I^{2}}{A_{1} k_{1}\left(T_{c}-T_{0}\right)}, \\
& D_{2}=\frac{L^{2} R_{2} I^{2}}{C_{R} A_{2} k_{1} L_{2}\left(T_{c}-T_{0}\right)}, \\
& F_{1}=1, \\
& F_{2}=\alpha_{R} .
\end{aligned}
$$

The parabolic heat diffusion equations are easily obtained by considering that for the classical heat diffusion equation the relaxation time disappears in (5a); that is, $\tau_{i}=0$ and only one initial condition on the temperature is needed to determine the temperature distribution inside the pellets.

\section{Thermal Field: Solution Method}

The analytical solution of (5a) may be obtained by using the SoV technique as in [13].

The hyperbolic temperature field for both the pellets is provided by (7a) and (8a). It is expressed as the sum of a steady state part $\vartheta_{\mathrm{ss}, i}(\xi)$ and of a complementary part:

$$
\begin{aligned}
& \vartheta_{1}(\xi, \eta)=\vartheta_{\mathrm{ss}, 1}(\xi) \\
& +\exp \left(-\frac{\eta}{2 \tau_{1}}\right) \sum_{n=1}^{\infty} A_{n, 1} \sin \left(\lambda_{n} \xi\right) \Psi_{n, 1}(\eta) \\
& +\exp \left(-\frac{\eta}{2 \tau_{1}}\right) \sum_{n=1}^{\infty} C_{n} D_{1} \sin \left(\lambda_{n} \xi\right) \zeta_{n, 1}(\eta), \\
& \vartheta_{\mathrm{ss}, 1}(\xi)=-\frac{D_{1}}{2} \xi^{2}+\left(h+\frac{D_{1}}{2}-1\right) \xi+1, \\
& \zeta_{n, 1}(\eta)=\frac{\sinh \left(\sqrt{\beta_{n, 1}^{2}-\lambda_{n}^{4}} \eta\right)}{\sqrt{\beta_{n, 1}^{2}-\lambda_{n}^{4}}}, \\
& \vartheta_{2}(\xi, \eta)=\vartheta_{\mathrm{ss}, 2}(\xi) \\
& +\exp \left(-\frac{\eta}{2 \tau_{2}}\right) \sum_{n=1}^{\infty} A_{n, 2} \sin \left(\lambda_{n} \xi\right) \Psi_{n, 2}(\eta) \\
& +\exp \left(-\frac{\eta}{2 \tau_{2}}\right) \sum_{n=1}^{\infty} C_{n} D_{2} \sin \left(\lambda_{n} \xi\right) \zeta_{n, 2}(\eta) \text {, } \\
& \vartheta_{\mathrm{ss}, 2}(\xi)=-\frac{D_{2}}{2 \alpha_{R}} \xi^{2}+\left(h+\frac{D_{2}}{2 \alpha_{R}}-1\right) \xi+1 \text {, } \\
& \zeta_{n, 2}(\eta)=\frac{\sinh \left(\sqrt{\beta_{n, 2}^{2}-\lambda_{n}^{4} \alpha_{R}^{2}} \eta\right)}{\sqrt{\beta_{n, 1}^{2}-\lambda_{n}^{4} \alpha_{R}^{2}}} .
\end{aligned}
$$

The eigenvalues $\lambda_{n}=n \pi$ are the same for both the semiconductors. Moreover,

$$
\begin{aligned}
\beta_{n, i} & =\lambda_{n}^{2} F_{i}-\frac{1}{2 \tau_{i}}, \\
\Psi_{n, i}(\eta) & =\cosh \left(\sqrt{\beta_{n, i}^{2}-\lambda_{n}^{4} F_{i}^{2}} \eta\right)+\frac{\zeta_{n, i}}{2 \tau_{i}}, \\
A_{n, i} & =\frac{2}{\lambda_{n}}\left[(-1)^{n} h+\frac{D_{i}\left[(-1)^{n}-1\right]}{\lambda_{n}^{2} F_{i}}-1\right], \\
C_{n} & =\frac{2\left[1-(-1)^{n}\right]}{\lambda_{n}} .
\end{aligned}
$$


The parabolic solution for both the semiconductors may be obtained as suggested in [14] and is expressed by

$$
\begin{aligned}
& \vartheta_{1}(\xi, \eta)=\vartheta_{\mathrm{ss}, 1}(\xi)+\sum_{n=1}^{\infty} A_{n, 1} \sin \left(\lambda_{n} \xi\right) \exp \left(-\lambda_{n}{ }^{2} \eta\right) \\
& \vartheta_{2}(\xi, \eta)=\vartheta_{\mathrm{ss}, 2}(\xi) \\
& \quad+\sum_{n=1}^{\infty} A_{n, 2} \sin \left(\lambda_{n} \xi\right) \exp \left(-\alpha_{R} \lambda_{n}{ }^{2} \eta\right) .
\end{aligned}
$$

The subscript $i$ always refers to the kind of semiconductor: $i=1$ for semiconductor $\mathrm{N}$ and $i=2$ for the $\mathrm{P}$ one.

\section{Heat Fluxes: Solution Method}

In order to complete the thermal analysis of the transient behaviour of the device, after obtaining the thermal fields, it is necessary to determine the conductive heat fluxes of each semiconductor. As regards the hyperbolic model, the thermal fluxes are given according to the non-Fourier heat equation, as in [15]. In fact the conductive heat flux for each pellet may be expressed as in

$$
q_{i}(x, t)+\bar{\tau}_{i} \frac{\partial q_{i}(x, t)}{\partial t}=-k_{i} A_{i} \frac{\partial T_{i}(x, t)}{\partial x}
$$

with the initial condition

$$
q_{i}(x, 0)=0 .
$$

By means of the dimensionless variables, listed in the Nomenclature, (11a) and (11b) may be rewritten as (12a) and (12b). Consider

$$
\begin{aligned}
Q_{i}(\xi, \eta)+\tau_{i} \frac{\partial Q_{i}(\xi, \eta)}{\partial \eta} & =-v_{i} \frac{\partial \vartheta_{i}(\xi, \eta)}{\partial \xi}, \\
Q_{i}(\xi, 0) & =0
\end{aligned}
$$

where $v_{1}=1$ for semiconductor $\mathrm{N}$ and $v_{2}=k_{R} A_{R}$ for semiconductor P. By integrating (12a) and by applying the initial condition, the hyperbolic thermal fluxes for each semiconductor are provided by

$$
\begin{aligned}
Q_{i} & (\xi, \eta) \\
& =-\left.\frac{v_{i}}{\tau_{i}} \exp \left(-\frac{\eta}{\tau_{i}}\right) \int_{0}^{\eta} \frac{\partial \vartheta_{i}(\xi, \omega)}{\partial \xi}\right|_{\xi} \exp \left(\frac{\omega}{\tau_{i}}\right) d \omega .
\end{aligned}
$$

As regards the parabolic heat fluxes, the relaxation time disappears in (11a) and each dimensionless conductive contribution may be expressed as in

$$
Q_{i}(\xi, \eta)=-v_{i} \frac{\partial \vartheta_{i}(\xi, \eta)}{\partial \xi}
$$

After evaluating the temperature gradient, according to (7a), it is substituted in (13). By integrating it and after some other calculations, the dimensionless hyperbolic conductive heat flux for each semiconductor is provided by (15) and (16). Consider

$$
\begin{aligned}
& Q_{1}(\xi, \eta)=\left[h+D_{1}\left(\frac{1}{2}-\xi\right)-1\right]\left(\exp \left(-\frac{\eta}{\tau_{1}}\right)-1\right) \\
& -\frac{\exp \left(-\eta / 2 \tau_{1}\right)}{\tau_{1}} \sum_{n=1}^{\infty} B_{n, 1} \cos \left(\lambda_{n} \xi\right) \zeta_{n, 1}(\eta)+\sum_{n=1}^{\infty} E_{n, 1}, \\
& Q_{2}(\xi, \eta)=v_{2}\left[h+\frac{D_{2}}{\alpha_{R}}\left(\frac{1}{2}-\xi\right)-1\right] \\
& \cdot\left(\exp \left(-\frac{\eta}{\tau_{2}}\right)-1\right)-v_{2} \frac{\exp \left(-\eta / 2 \tau_{2}\right)}{\tau_{2}} \sum_{n=1}^{\infty} B_{n, 2} \\
& \cdot \cos \left(\lambda_{n} \xi\right) \zeta_{n, 2}(\eta)+v_{2} \sum_{n=1}^{\infty} E_{n, 2}, \\
& B_{n, i}=A_{n, i} \lambda_{n}+C_{n} \frac{D_{i}}{2 \lambda_{n} F_{i}}, \\
& E_{n, i}=\frac{C_{n} D_{i} \cos \left(\lambda_{n} \xi\right)}{\lambda_{n} F_{i}} \exp \left(-\frac{\eta}{2 \tau_{i}}\right) \\
& \cdot \cosh \left(\sqrt{\beta_{n, i}^{2}-\lambda_{n}^{4} F_{i}^{2}} \eta\right)-\frac{C_{n} D_{i} \cos \left(\lambda_{n} \xi\right)}{\lambda_{n} F_{i}} \\
& \left.\tau_{i}\right) \cdot
\end{aligned}
$$

\section{Performances of the Microcooler}

The cooling and the heating load effects are expressed by the thermal fluxes $Q_{c}$ and $Q_{h}$, respectively. They may be written in dimensionless terms by considering an energy balance for the hot and the cold junctions, as suggested by

$$
\begin{aligned}
& Q_{c}=G+Q_{1, c}(0, \eta)+Q_{2, c}(0, \eta), \\
& Q_{h}=M+Q_{1, h}(1, \eta)+Q_{2, h}(1, \eta) .
\end{aligned}
$$

The dimensionless terms $Q_{i, j}$ represent the conductive heat fluxes for semiconductors $\mathrm{N}$ and $\mathrm{P}$, provided, in the hyperbolic case, by (15) and (16) and evaluated at the cold and the hot ends, $\xi=0$ and $\xi=1$, respectively. $G$ and $M$ represent the dimensionless contributions due to the Peltier effect and they are defined in the Nomenclature. In particular, $G$ is the thermal power absorbed at the cold junction and $M$ is the thermal power released at the hot junction.

In the parabolic model (21a) and (21b) has to be considered as follows:

$$
\begin{aligned}
& Q_{c}=G-\frac{\partial \vartheta_{1}(0, \eta)}{\partial \xi}-k_{R} A_{R} \frac{\partial \vartheta_{2}(0, \eta)}{\partial \xi}, \\
& Q_{h}=M-\frac{\partial \vartheta_{1}(1, \eta)}{\partial \xi}-k_{R} A_{R} \frac{\partial \vartheta_{2}\left(L_{R}, \eta\right)}{\partial \xi} .
\end{aligned}
$$

It can be seen that the cooling load and the thermal power released at the hot junction, provided by (19)-(21b), are valid 


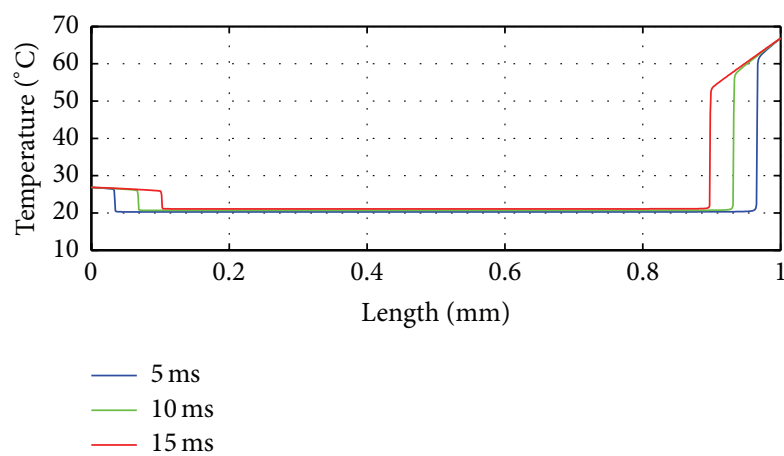

(a)

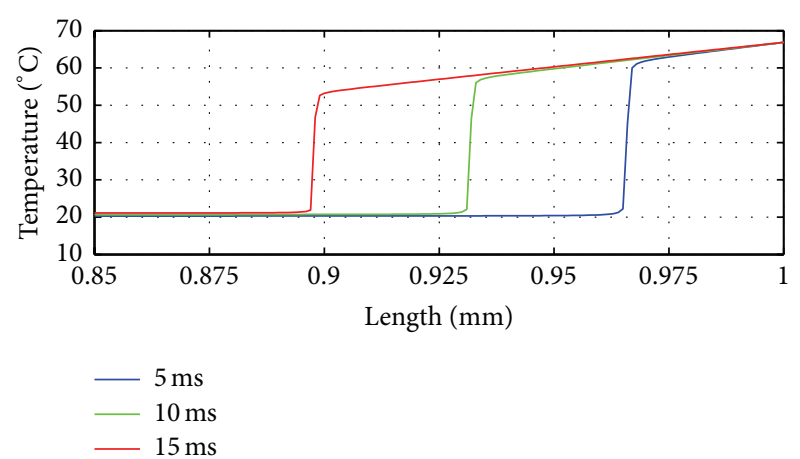

(b)

Figure 2: Temperature profiles for semiconductor $\mathrm{N}$ for early time values (a). Enlargement of the wave front (b). Relaxation time $=20$ ms.

for a single thermoelectric pair. In the case of $n$-pairs, it is sufficient to multiply the above mentioned equations for the number of pairs. The performances of a thermoelectric cooler are evaluated by means of the coefficient of performance, COP, defined in

$$
\mathrm{COP}=\frac{Q_{c}}{Q_{h}-Q_{c}} .
$$

\section{Results and Discussion}

A miniature thermoelectric cooler as in $[4,10]$ has been considered for the simulations. The geometrical characteristics and some properties of both the semiconductors are listed in Table 1. As regards the thermoelectric materials, $\mathrm{Bi}_{2}\left(\mathrm{Te}_{0.94} \mathrm{Se}_{0.06}\right)_{3}$ and $\left(\mathrm{Bi}_{0.25} \mathrm{Sb}_{0.75}\right) \mathrm{Te}_{3}$ are selected for the $\mathrm{N}$ and P-type semiconductors, respectively, as in $[4,10]$. The temperature $T_{c}$ of the cold end of the semiconductors is set at $27^{\circ} \mathrm{C}$, whereas the hot ends temperature is set at $T_{h}=67^{\circ} \mathrm{C}$ as in [10], where the temperatures are given in Kelvin. The working current is assumed to be $1 \mathrm{~A}$. The initial temperature is set at $20^{\circ} \mathrm{C}$ as in [10].

In order to consider the thermal conductivity, the Seebeck coefficient, and the electrical resistivity of the above mentioned materials, a quadratic relationship, described by (23), is employed. Consider

$$
p_{j}=p_{j}\left(T_{\mathrm{ref}}\right)\left[1+a_{j}\left(T-T_{\mathrm{ref}}\right)+b_{j}\left(T-T_{\mathrm{ref}}\right)^{2}\right],
$$

where $p_{j}$ indicates the generic property, that is, $k, S$, and $\rho_{e}$, which is evaluated, in the present paper at an average temperature $T_{\text {avg }}=\left(T_{h}+T_{c}\right) / 2 . T_{\text {ref }}$ is provided in [10] and the coefficients $a_{j}$ and $b_{j}$ are listed in $[4,10]$. The subscript $j=1$ is used for the thermal conductivity, $j=2$ is used for the Seebeck coefficient, and $j=3$ is used for the electrical resistivity. The relaxation time value considered for the simulations of the hyperbolic thermal behaviour is $20 \mathrm{~ms}$.

Figures 2 and 3 show the temperature profiles in semiconductor $\mathrm{N}$ for different time values. The wave fronts travel in the material at a speed of $\sqrt{\alpha / \bar{\tau}}$.

During their motion, these wave fronts give constructive interference and then they are reflected by the opposite end of the semiconductor.
TABLE 1: Geometrical sizes and properties of the semiconductors.

\begin{tabular}{lccc}
\hline Parameter & N-type & P-type & Reference \\
\hline$L(\mathrm{~m})$ & $1 \times 10^{-3}$ & $1 \times 10^{-3}$ & {$[4,10]$} \\
$A\left(\mathrm{~m}^{2}\right)$ & $0.25 \times 10^{-6}$ & $0.25 \times 10^{-6}$ & {$[4,10]$} \\
$\rho\left(\mathrm{kg} / \mathrm{m}^{3}\right)$ & 10922.08 & 10922.08 & {$[3]$} \\
$c(\mathrm{~J} / \mathrm{kg} \mathrm{K})$ & 160 & 190 & {$[3]$} \\
\hline
\end{tabular}

By the observation of the plotted temperature profiles, it is evident that for early time values the temperature profiles have oscillations at the wave fronts.

This phenomenon is due to the numerical instability caused by the impossibility of representing the infinite terms in the series in (7a) and (8a). To remove this numerical instability, the Separation of Variables Method, which has been used here for solving the hyperbolic equation and coming from [13], should be replaced by a different approach. For example, we might use a finite series solution at early times coming from the combination of Laplace transform and method of images (e.g., sources and sinks) as given in [16]. But this is a subject for future research.

It is worth noting that the above mentioned oscillations are more evident in semiconductor P, Figures 4 and 5, due to the different diffusivity of this pellet, more precisely, because of the presence of the thermal diffusivity ratio in (8a).

For time values higher than $230 \mathrm{~ms}$, the hyperbolic temperature field becomes similar to the parabolic one and the oscillations are not so marked as in the early times as depicted in Figures 3 and 5.

Figure 6 shows the influence of the relaxation time on the temperature profiles for semiconductor $\mathrm{N}$. It is clear that if the relaxation time decreases, the temperature profile becomes similar to the parabolic one, as the speed of the thermal wave in the semiconductor increases. Moreover, at the thermal wave regions, a larger relaxation time value affects the term $\tau_{i} D_{i} \delta(\eta)$ significantly in (5a). The conductive heat flux in semiconductor N, provided by (15) and evaluated at the cold end $\xi=0$, is represented in Figure 7(a). Some discontinuity points are evident in the plot of the hyperbolic contribution whose enlargement is given by Figure 7(b). 


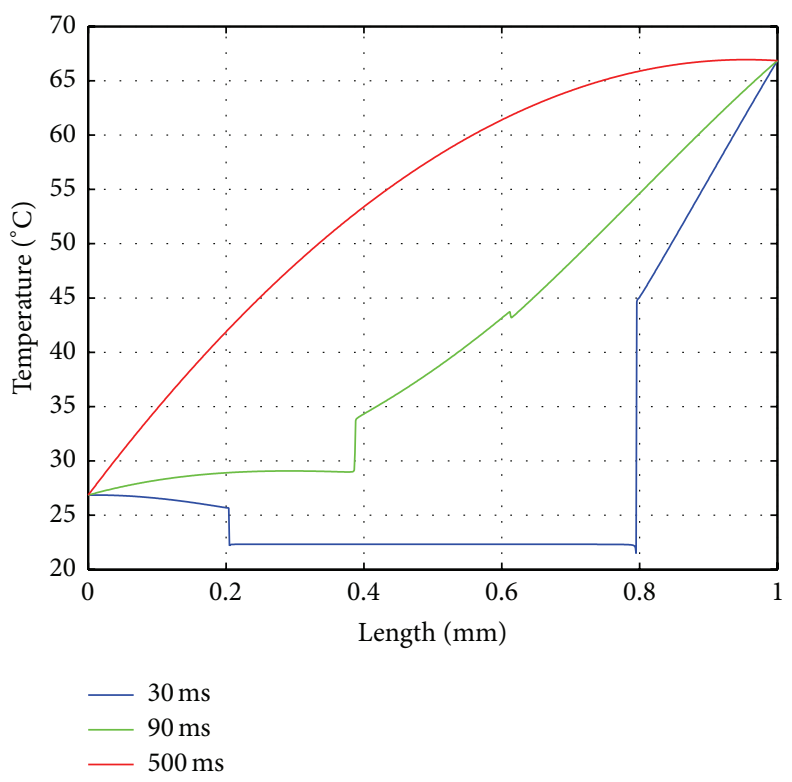

FIgURE 3: Temperature profiles for semiconductor N (high time values). Relaxation time $=20 \mathrm{~ms}$.

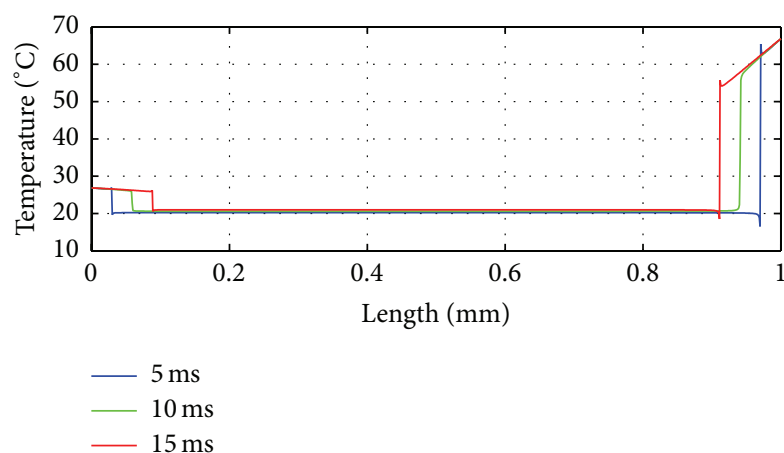

(a)

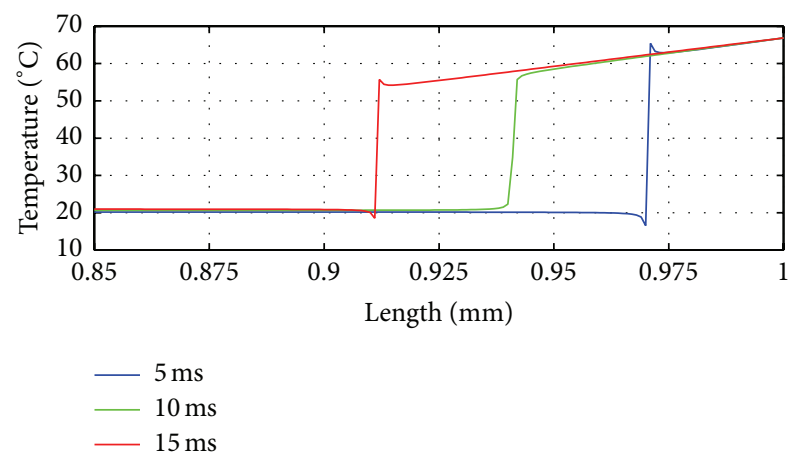

(b)

FIGURE 4: Temperature profiles for semiconductor P (early time values). Relaxation time $=20 \mathrm{~ms}$.

This phenomenon takes place because each reflection of the wave front produces discontinuities in the heat flux. For early time values, the parabolic model does not allow the heat flux at the cold end to be properly represented. In fact, it goes to infinity when the time approaches zero. For time values higher than $500 \mathrm{~ms}$ the hyperbolic and the parabolic heat fluxes tend to overlap.

In Figure 8 the conductive heat flux at the hot end of semiconductor $\mathrm{N}$ has been depicted.

Figure 9 shows the cooling load profile, whose analytical expression is provided by (19). In comparison with Figure 7 , the cooling load shows further discontinuities due to the contributions of the wave fronts due to the second semiconductor (the $\mathrm{P}$ one).

This occurs because the two wave fronts travel at different speeds, due to the different thermal diffusivities. It has to be observed that the value of the cooling load at $t=0$ is not zero, because of the contribution of the Peltier effect.

As regards the COP, it is plotted for time values higher than $200 \mathrm{~ms}$. In fact, for earlier time values, the employed model, based on imposed time-independent boundary temperatures, does not allow us to describe accurately the real thermal behaviour of the device: the thermal power released at the hot junction and the cooling load cross as depicted in Figure 10, providing a zero denominator in (22).

As the time increases, the hyperbolic COP is similar to the parabolic one, reaching the value of about 0.72 as shown in Figure 11.

Figure 12 shows the COP profile as a function of the time for different values of the parameter $F_{2}$ (thermal diffusivity ratio). By increasing this ratio, it can be observed that the COP increases at large time values. In Figure 13 the COP behaviour as a function of the time is plotted for different values of the parameter $D_{1}$. This parameter expresses the dimensionless internal heat generation due to the Joule effect. By increasing $D_{1}$, the volumetric effects, due to the heat dissipation, become more and more relevant, if compared with the Peltier heat, which is a superficial effect. This phenomenon causes a sensible reduction of the cooling load, 


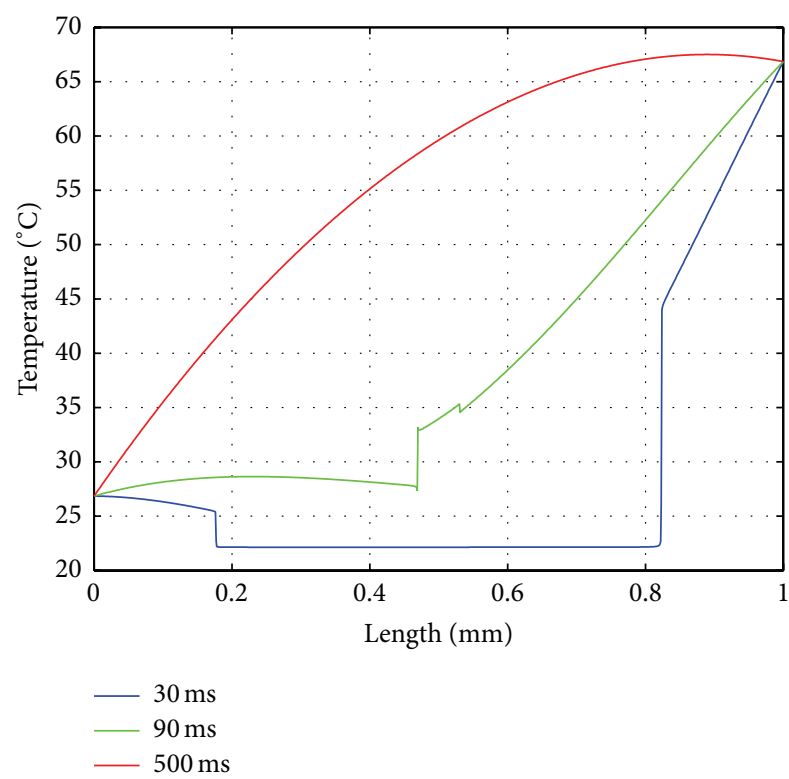

FIgURE 5: Temperature profiles for semiconductor P (high time values). Relaxation time $=20 \mathrm{~ms}$.

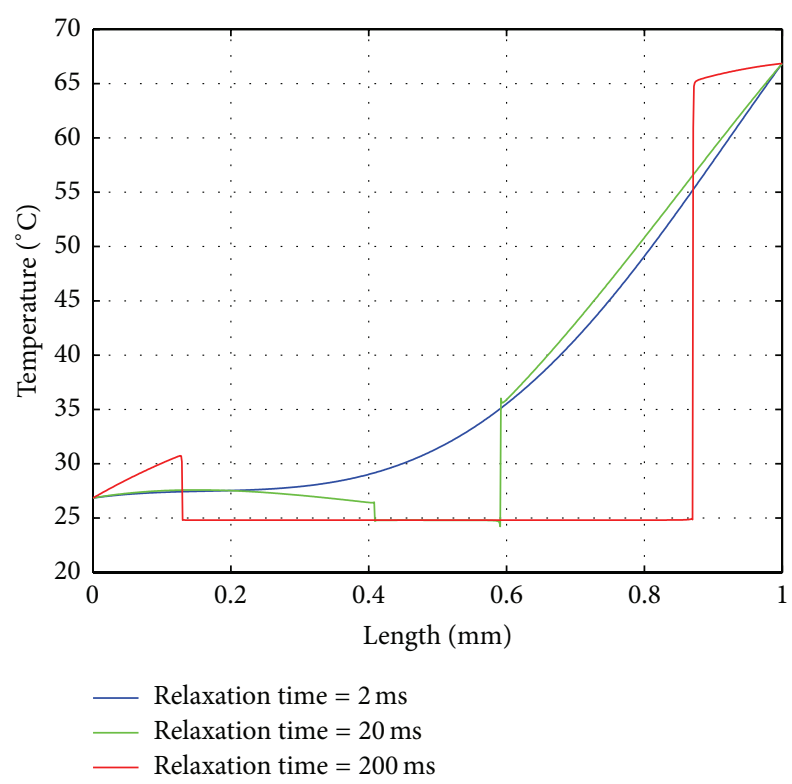

FIGURE 6: Influence of the relaxation time on the temperature profile for semiconductor $\mathrm{N}$ when $t=60 \mathrm{~ms}$.

which affects the COP. The parameter $D_{2}$ has the same influence on the COP, as the one depicted in Figure 13.

Moreover, as regards the COP, the values deriving from the present analysis under steady state conditions are in agreement with those presented in the numerical parabolic coupled model available in the literature [10], as shown in Table 2. However, by applying an electric current of $1.5 \mathrm{~A}$, it can be observed that the COP value under steady state condition is noticeably higher than the value presented in
TABLE 2: Comparison of the COP values under steady state conditions.

\begin{tabular}{lccc}
\hline$I(\mathrm{~A})$ & Present & Reference [10] & Reference [4] \\
\hline 0.6 & 1 & 0.98 & - \\
0.9 & 0.80 & 0.80 & - \\
1.2 & 0.56 & 0.60 & - \\
1.5 & 0.45 & 0.43 & 0.22 \\
\hline
\end{tabular}

the numerical dynamic parabolic coupled model [4]. This is due to the more realistic time-dependent boundary conditions employed in the simulations.

It is worth noting that in the coupled numerical parabolic model [4] the steady state is reached after about $600 \mathrm{~s}$ due to the higher thermal inertia of the system. Therefore, the future commitment is focussed on the hyperbolic modelling developed by means of the more realistic time-dependent boundary conditions of the first kind. This is done by considering the presence of the heat sink at the hot end of the system.

\section{Conclusion}

An analytical development of the hyperbolic behaviour of a micro thermoelectric cooler has been presented in which the sudden application of the electric current is considered by means of the Heaviside function. The thermal fields, obtained by means of the Separation of Variables technique with imposed boundary conditions of the first kind, show oscillations by the wave fronts for very early times, which are more marked for semiconductor $\mathrm{P}$. The reflection of the wave fronts of both the semiconductors yields discontinuities in the cooling load profiles.

Moreover, the not realistic imposed time-independent boundary conditions of the first kind allow us to represent the COP profile only for time values higher than $0.2 \mathrm{~s}$, as the intersection of the thermal power released at the hot junction and the cooling load occurs.

The future commitment is focussed on the hyperbolic modelling developed by means of the more realistic timedependent boundary conditions of the first kind. This is done by considering the presence of the heat sink at the hot end of the system.

\section{Nomenclature (Symbol, Quantity, and SI Unit)}

A: Cross-sectional area, $\mathrm{m}^{2}$

$A_{R}$ : Cross-sectional area ratio $A_{R}=A_{2} / A_{1}$, -

c: $\quad$ Specific heat capacity, $\mathrm{J} / \mathrm{kg} \mathrm{K}$

$C_{R}:$ Heat capacity ratio, $C_{R}=\varrho_{2} c_{2} /\left(\varrho_{1} c_{1}\right),-$

$e: \quad$ Electronic charge, $\mathrm{C}$

G: Coefficient

$G=\left[\left(S_{2}-S_{1}\right) /\left(T_{c}-T_{0}\right) k_{1} A_{1}\right] T_{c} L I,-$

$h: \quad$ Temperature ratio, $h=\left(T_{h}-T_{0}\right) /\left(T_{c}-T_{0}\right),-$

$H($ ): Heaviside function, - 


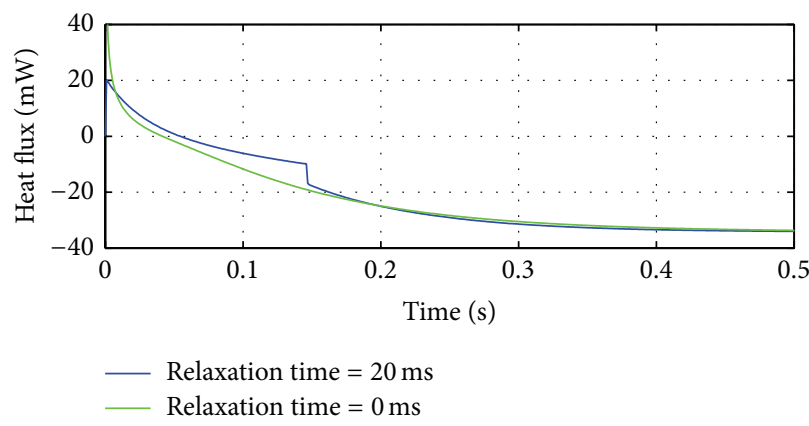

(a)

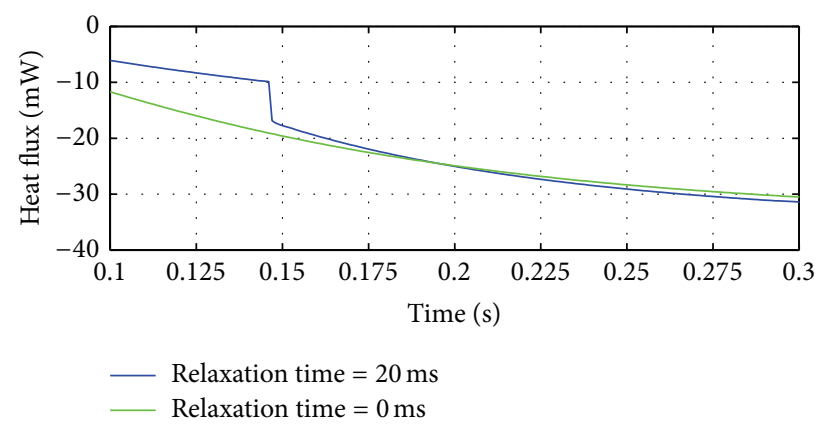

(b)

FIGURE 7: Conductive heat flux at the cold end of semiconductor N (a). Enlargement of the discontinuities (b).

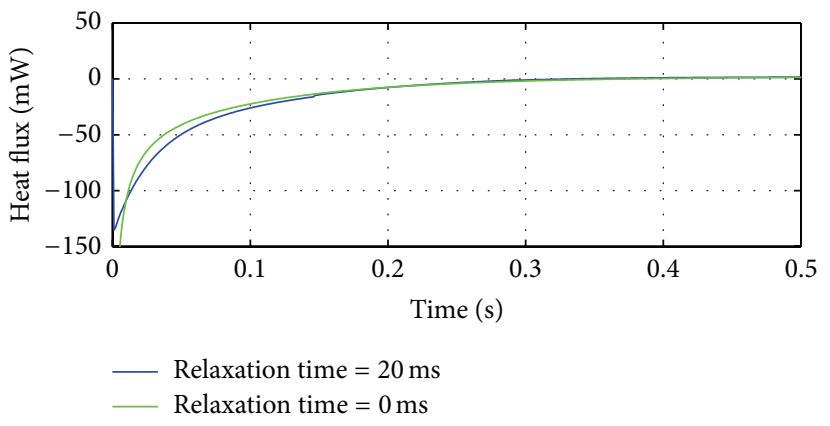

(a)

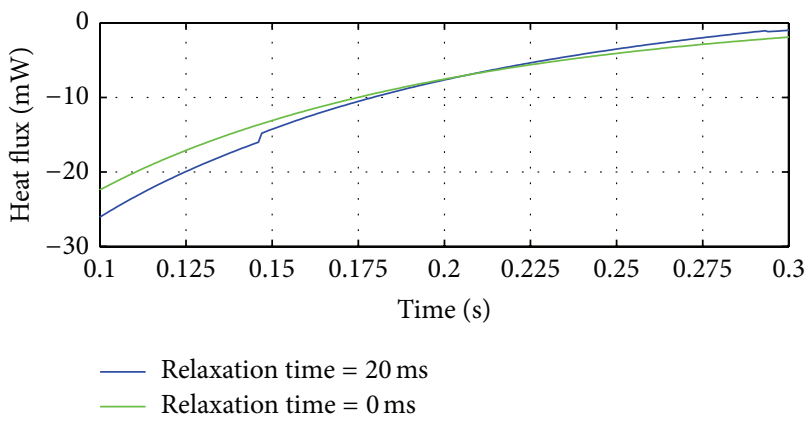

(b)

FIgUre 8: Conductive heat flux at the hot end of semiconductor N (a). Enlargement of the discontinuities (b).

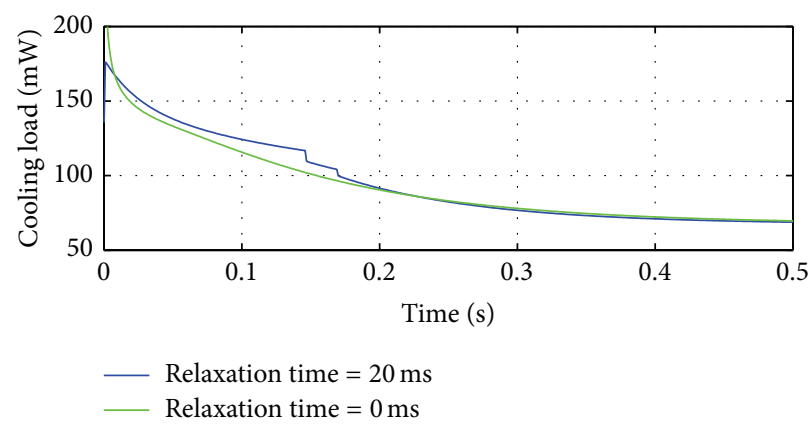

(a)

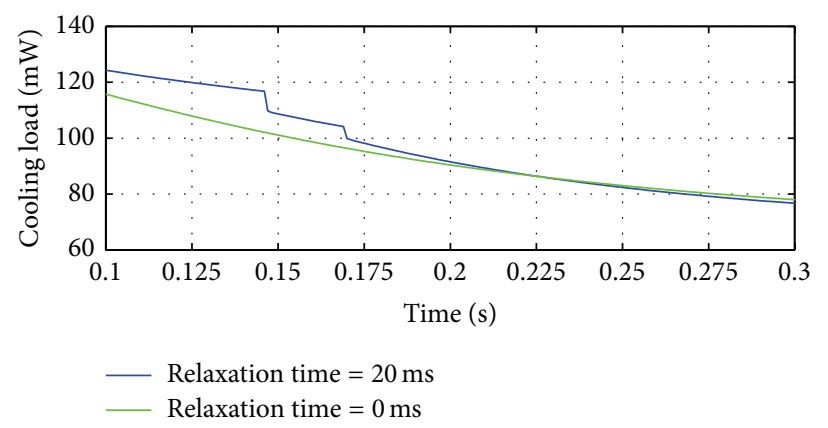

(b)

Figure 9: Cooling load (a). Enlargement of the discontinuities (b).

I: $\quad$ Electric current, A

$J$ : Electric current density, $\mathrm{A} / \mathrm{m}^{2}$

$k$ : Thermal conductivity, $\mathrm{W} / \mathrm{m} \mathrm{K}$

$k_{R}$ : Thermal conductivity ratio, $k_{R}=k_{2} / k_{1},-$

$L$ : Length of the components, $\mathrm{m}$

$M$ : Coefficient $M=\left[\left(S_{2}-S_{1}\right) /\left(T_{c}-\right.\right.$ $\left.\left.T_{0}\right) k_{1} A_{1}\right] T_{h} L I,-$

$q$ : Heat flux, $\mathrm{W}$

$q^{*}:$ Specific heat flux, $\mathrm{W} / \mathrm{m}^{2}$

$q_{c}$ : Cooling load effect, $\mathrm{W}$

$q_{h}$ : Thermal power released at the hot junction, $\mathrm{W}$
$Q_{c}: \quad$ Dimensionless cooling effect, $Q_{c}=q_{c} L /\left[k_{1} A_{1}\left(T_{c}-\right.\right.$ $\left.\left.T_{0}\right)\right],-$

$Q_{h}$ : Dimensionless thermal power released at the hot junction, $Q_{h}=q_{h} L /\left[k_{1} A_{1}\left(T_{c}-T_{0}\right)\right]$, -

$R: \quad$ Electrical resistance, $R=\varrho_{e} L / A, \Omega$

$S: \quad$ Seebeck coefficient, $\mathrm{V} / \mathrm{K}$

$t: \quad$ Time, $\mathrm{s}$

$T: \quad$ Temperature, ${ }^{\circ} \mathrm{C}$

$T_{\text {avg }}$ : Average temperature $T_{\text {avg }}=\left(T_{h}+T_{c}\right) / 2,{ }^{\circ} \mathrm{C}$

$T_{c}$ : Cold junction temperature, ${ }^{\circ} \mathrm{C}$

$T_{h}$ : Hot junction temperature, ${ }^{\circ} \mathrm{C}$ 


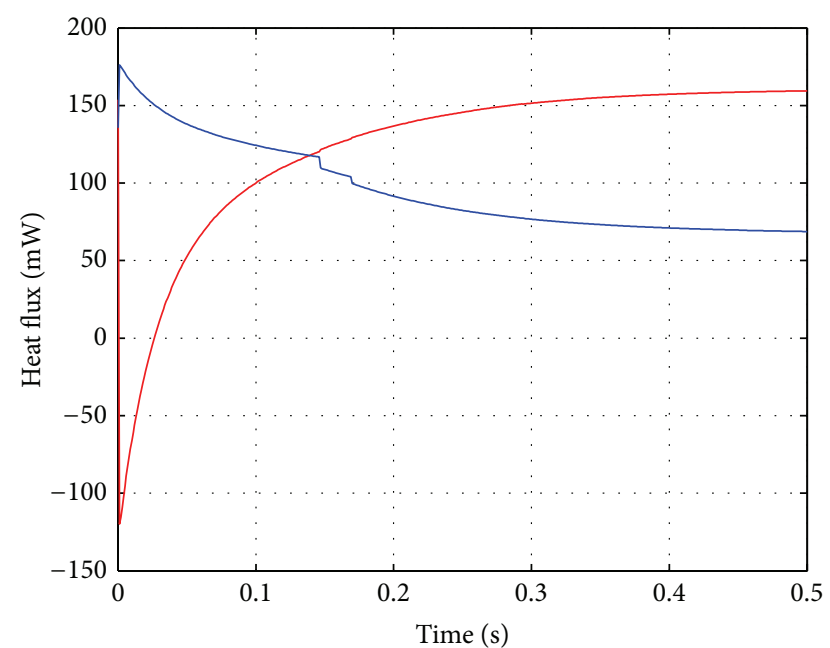

Thermal power released at the hot junction Cooling load-relaxation time: $20 \mathrm{~ms}$

FIgURE 10: Thermal power released at the hot junction and cooling load. Relaxation time $20 \mathrm{~ms}$.

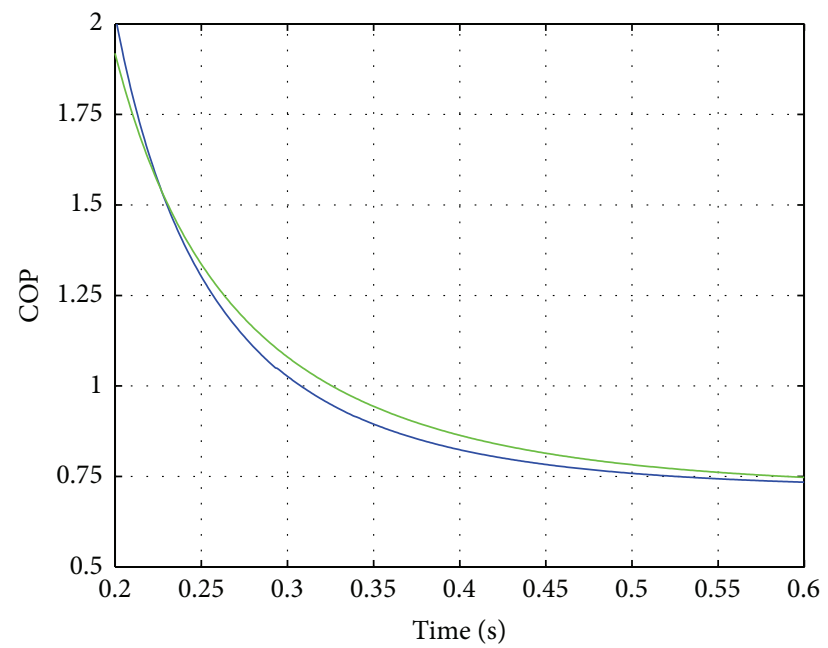

Relaxation time: $20 \mathrm{~ms}$

Relaxation time: $0 \mathrm{~ms}$

Figure 11: COP as a function of time.

$T_{\text {ref }}:$ Reference temperature, ${ }^{\circ} \mathrm{C}$

$T_{0}$ : Initial temperature, ${ }^{\circ} \mathrm{C}$

$x$ : Spatial coordinate, -

$\alpha$ : Thermal diffusivity, $\mathrm{m}^{2} / \mathrm{s}$

$\alpha_{R}$ : Thermal diffusivity ratio, $\alpha_{R}=\alpha_{2} / \alpha_{1}$, -

$\delta($ ): Dirac's Delta function, -

$\eta$ : Dimensionless time, $\eta=\alpha t / L^{2},-$

$\vartheta$ : Dimensionless temperature, $\mathcal{\vartheta}=(T-$ $\left.T_{0}\right) /\left(T_{c}-T_{0}\right),-$

$v_{i}: \quad$ Coefficient, $v_{i}=k_{i} A_{i} /\left(k_{1} A_{1}\right)$

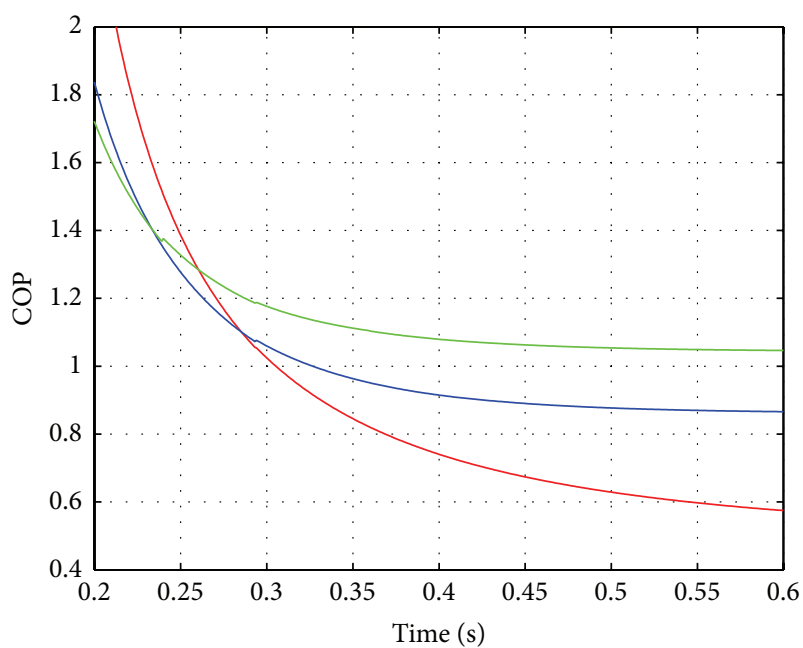

$\begin{aligned}-F_{2} & =0.5 \\ -F_{2} & =1 \\ F_{2} & =1.5\end{aligned}$

FIGURE 12: COP as a function of time for different values of $F_{2}$.

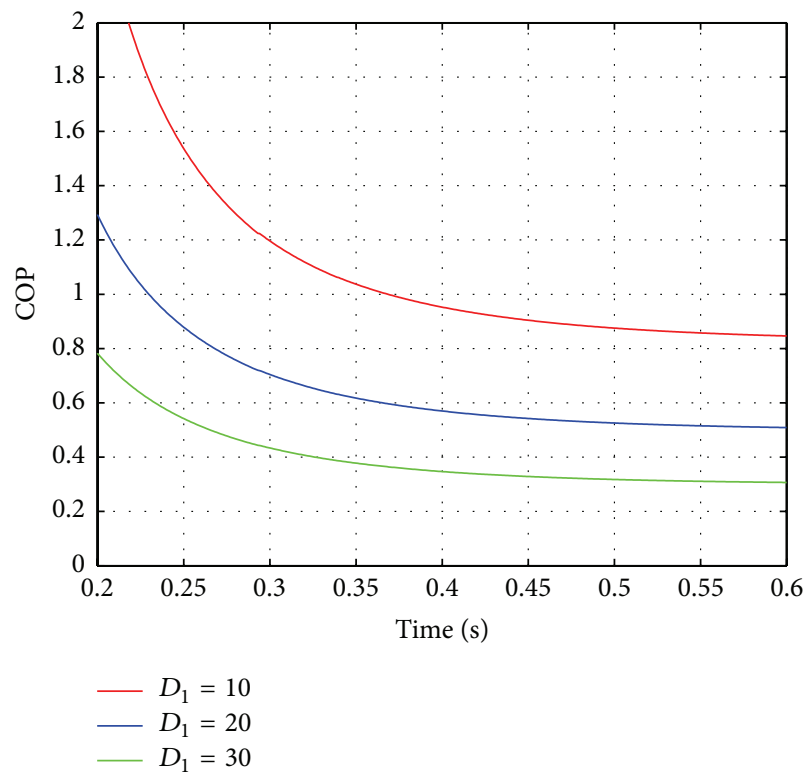

FIgURE 13: COP as a function of time for different values of $D_{1}$.

$\xi: \quad \xi=x / L$, dimensionless space, -

$\rho:$ Material density, $\mathrm{kg} / \mathrm{m}^{3}$

$\rho_{e}$ : Electrical resistivity, $\Omega \mathrm{m}$

$\bar{\tau}$ : Relaxation time, $\mathrm{s}$

$\tau$ : Dimensionless relaxation time, $\tau=\alpha \bar{\tau} / L^{2}$,

Ф: Electrostatic potential, V.

Subscripts

$i$ : $i$ th semiconductor (1 or 2 ). 


\section{Conflict of Interests}

The authors declare that there is no conflict of interests regarding the publication of this paper.

\section{References}

[1] M. Naji, M. Alata, and M. A. Al-Nimr, "Transient behaviour of a thermoelectric device," Proceedings of the Institution of Mechanical Engineers A: Journal of Power and Energy, vol. 217, no. 6, pp. 615-621, 2003.

[2] C.-H. Cheng, S.-Y. Huang, and T.-C. Cheng, "A threedimensional theoretical model for predicting transient thermal behavior of thermoelectric coolers," International Journal of Heat and Mass Transfer, vol. 53, no. 9-10, pp. 2001-2011, 2010.

[3] C.-H. Cheng and S.-Y. Huang, "Development of a non-uniformcurrent model for predicting transient thermal behavior of thermoelectric coolers," Applied Energy, vol. 100, pp. 326-335, 2012.

[4] J.-H. Meng, X.-D. Wang, and X.-X. Zhang, "Transient modeling and dynamic characteristics of thermoelectric cooler," Applied Energy, vol. 108, pp. 340-348, 2013.

[5] G. De Aloysio and F. de Monte, "Thermal characterization of micro thermoelectric coolers: an analytical study," Journal of Physics: Conference Series, vol. 547, no. 1, Article ID 012007, 2014.

[6] M. Alata, M. A. Al-Nimr, and M. Naji, "Transient behavior of a thermoelectric device under the hyperbolic heat conduction model," International Journal of Thermophysics, vol. 24, no. 6 , pp. 1753-1768, 2003.

[7] R. Palma, J. L. Pérez-Aparicio, and R. L. Taylor, "Non-linear finite element formulation applied to thermoelectric materials under hyperbolic heat conduction model," Computer Methods in Applied Mechanics and Engineering, vol. 213-216, pp. 93-103, 2012.

[8] A. Figueroa and F. Vázquez, "Optimal performance and entropy generation transition from micro to nanoscaled thermoelectric layers," International Journal of Heat and Mass Transfer, vol. 71, pp. 724-731, 2014.

[9] R. Yang, G. Chen, A. R. Kumar, G. J. Snyder, and J.-P. Fleurial, "Transient cooling of thermoelectric coolers and its applications for microdevices," Energy Conversion and Management, vol. 46, no. 9-10, pp. 1407-1421, 2005.

[10] X. D. Wang, Y. X. Huang, C. H. Cheng, D. T. W. Lin, and C. H. Kang, "A three-dimensional numerical modeling of thermoelectric device with consideration of coupling of temperature field and electric potential field," Energy, vol. 47, no. 1, pp. 488497, 2012.

[11] D. Zhao and G. Tan, "A review of thermoelectric cooling: materials, modeling and applications," Applied Thermal Engineering, vol. 66, no. 1-2, pp. 15-24, 2014.

[12] R. Chein and G. Huang, "Thermoelectric cooler application in electronic cooling," Applied Thermal Engineering, vol. 24, no. 1415, pp. 2207-2217, 2004.

[13] A. Haji-Sheikh and J. V. Beck, "Green's function solution for thermal wave equation in finite bodies," International Journal of Heat and Mass Transfer, vol. 37, no. 17, pp. 2615-2626, 1994.

[14] M. N. Ozisik, Heat Conduction, Wiley, New York, NY, USA, 2nd edition, 1993.
[15] D. Y. Tzou, Macro- to Microscale Heat Transfer: The Lagging Behavior, Taylor \& Francis, Washington, DC, USA, 1996.

[16] A. Haji-Sheikh, F. de Monte, and J. V. Beck, "Temperature solutions in thin films using thermal wave Green's function solution equation," International Journal of Heat and Mass Transfer, vol. 62, no. 1, pp. 78-86, 2013. 


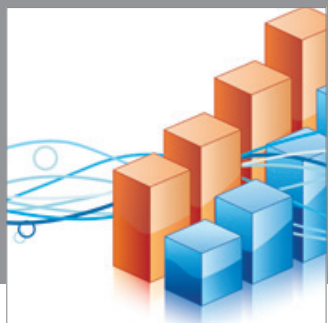

Advances in

Operations Research

mansans

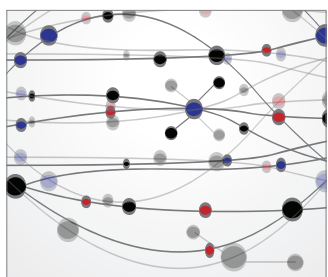

The Scientific World Journal
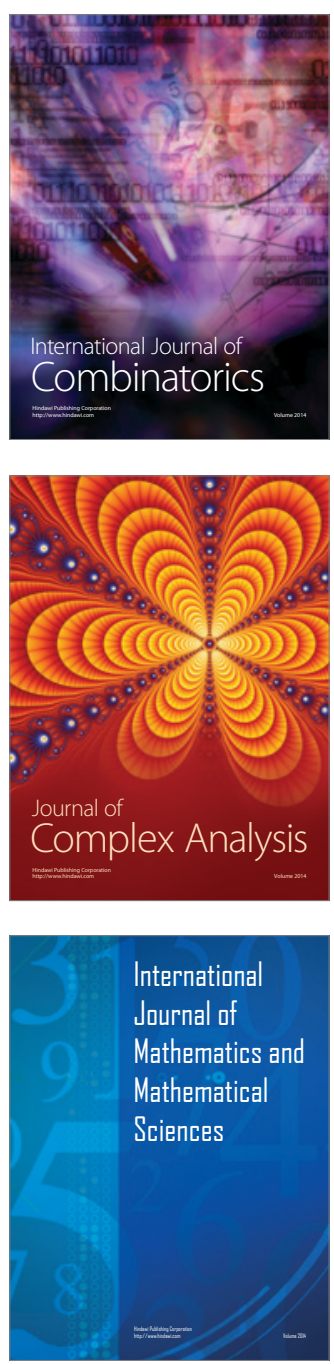
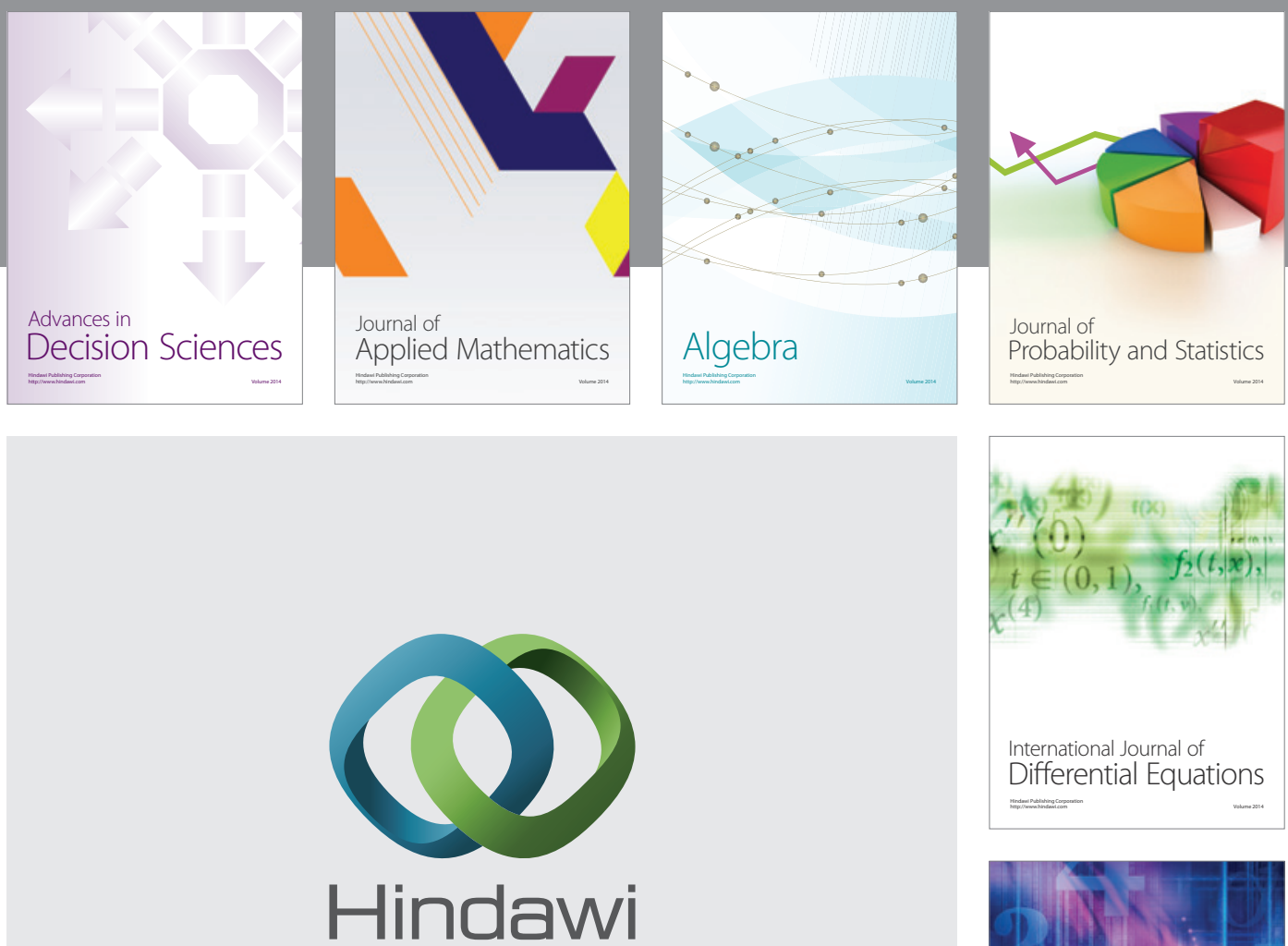

Submit your manuscripts at http://www.hindawi.com
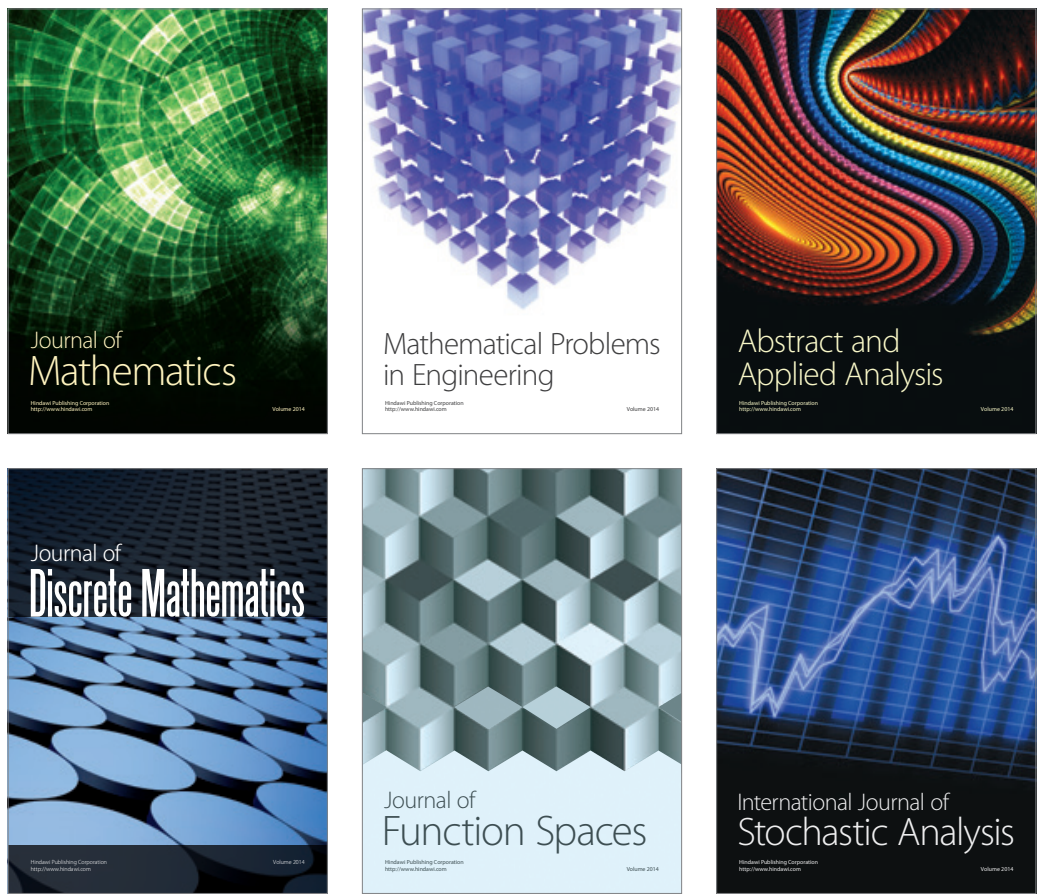

Journal of

Function Spaces

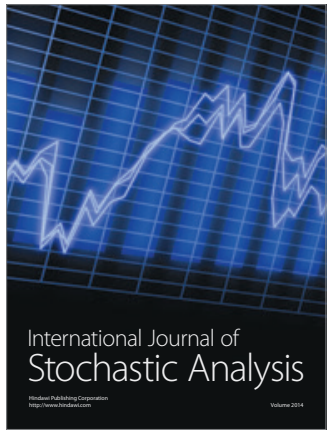

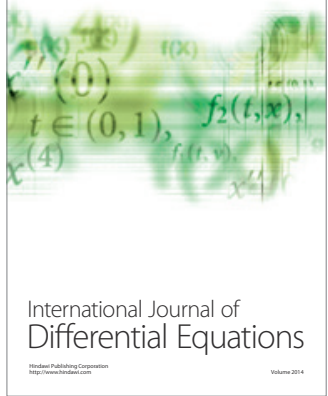
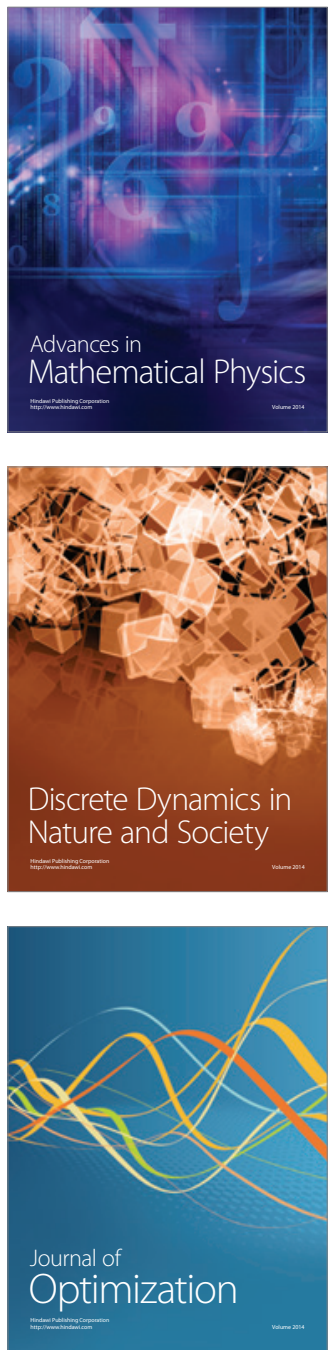